\title{
MODELAGEM MATEMÁTICA PARA SELEÇÃO DE CONJUNTOS MECANIZADOS AGRÍCOLAS PELO MENOR CUSTO OPERACIONAL
}

\author{
FÁBIO H. R. BAIO ${ }^{1}$, ANDREW D. RODRIGUES ${ }^{2}$, GILSON S. DOS SANTOS ${ }^{3}$, \\ SIMONE P. DA SILVA ${ }^{4}$
}

\begin{abstract}
RESUMO: A seleção de uma máquina agrícola pode tornar-se uma tarefa árdua, pois há diversas variáveis que devem ser consideradas. A escolha do equipamento mais adequado para uma propriedade agrícola é uma das etapas mais importantes do processo produtivo. O objetivo deste trabalho foi desenvolver um modelo de computador por programação linear em plataforma web para seleção automatizada de conjuntos mecanizados agrícolas, baseados no menor custo operacional. O programa, desenvolvido em linguagem ASP.NET, pode ser acessado gratuitamente pela Internet (www.maquinas.ufms.br). O usuário pode selecionar um conjunto mecanizado agrícola dentro de uma vasta lista de opções que contém suas especificações técnicas ou deixar que o programa lhe retorne automaticamente a melhor opção, pelo menor custo operacional. $\mathrm{O}$ programa desenvolvido proporciona ao usuário uma seleção racional via Internet de conjuntos mecanizados, permitindo o estudo econômico do uso das máquinas e implementos, sem a necessidade da instalação de programas dedicados no computador, que dificultariam a manutenção do banco de dados.
\end{abstract}

PALAVRAS-CHAVE: modelagem computacional, programação linear, seleção de máquinas.

\section{MATHEMATICAL MODELING TO SELECT MECHANIZED AGRICULTURAL SYSTEMS BY THE LOWEST OPERATIONAL COST}

\begin{abstract}
The selection of an agricultural machine may become a challenging task, because there are several variables that must be considered. Choosing the more suitable equipment to a farm is one of the most important production process steps. The purpose of this study was to develop a computational model by linear programming based on web platform to select automatically mechanized agricultural systems founded on the lowest operational cost. The software was developed in ASP.NET language and can be accessed for free by the Internet (www.maquinas.ufms.br). The user can select a mechanized agricultural system in a list that contains the specifications or let the system returns automatically the best option at the lowest operational cost. The developed software brought to the user via Internet a rational selection of mechanized systems, allowing the economic machinery studies, without any dedicated setup on the user's computer, which would hinder the database maintenance.
\end{abstract}

KEY WORDS: computer modeling, linear programing, machinery selection.

\section{INTRODUÇÃO}

A seleção de uma máquina agrícola pode tornar-se uma tarefa árdua, pois há diversas variáveis que devem ser consideradas. A escolha do equipamento mais adequado para uma propriedade agrícola é uma das etapas mais importantes do processo produtivo. A correta adequação da capacidade da máquina em relação à área é de extrema importância, pois afeta diretamente os custos produtivos. Modelos matemáticos podem ser considerados como ferramentas úteis na seleção de um maquinário agrícola.

\footnotetext{
${ }^{1}$ Professor da UFMS, Câmpus de Chapadão do Sul, Caixa Postal 112, Chapadão do Sul - MS, fabiobaio@ufms.br.

${ }^{2}$ Agronomia, UFMS, IC CNPq, andrew@agronomo.eng.br.

${ }^{3}$ Professor do IFMS, Câmpus de Coxim - MS, gilsonsatu@gmail.com.

${ }^{4}$ Professor da UFMS, Câmpus de Chapadão do Sul, simone.pereira@ufms.br.

Recebido pelo Conselho Editorial em: 16-1-2012

Aprovado pelo Conselho Editorial em: 26-9-2012
} 
Para MERCANTE et al. (2010), a mecanização agrícola está em fluxo contínuo de desenvolvimento e de criações de novas tecnologias; isso exige o uso racional dela, objetivando alcançar um maior rendimento e produção com menor gasto, como pode ser obtido com a utilização da informática na agricultura.

O termo modelo é normalmente utilizado para caracterizar estruturas que representam feições e objetos ou cenários. Como explana BAIO et al. (2004), um modelo matemático é uma estrutura que procura representar a realidade simplificadamente. Muitas vezes, os modelos matemáticos são elaborados objetivando a escolha de uma solução ótima. Uma solução ótima é uma solução viável que possui o valor mais favorável para a função objetivo, dependendo de se o objetivo é maximizar ou minimizar a função. A obtenção da solução ótima é dependente das variáveis de decisão, as quais podem estar sujeitas a uma série de restrições ou limitações. De acordo com BORNSTEIN et al. (2011), a programação linear é uma ferramenta para o planejamento de atividades para a obtenção de um resultado ótimo, respeitando as alternativas viáveis. Em um problema de programação linear, há algumas soluções viáveis, quando todas as restrições são satisfeitas, mas geralmente, há somente uma solução ótima, sendo uma exceção quando se encontram soluções infinitas. Há alguns problemas reais onde a solução somente faz sentido se as variáveis de decisão possuírem valores inteiros, como na seleção de máquinas agrícolas.

CENTENO \& KAERCHER (2010) relatam que os custos referentes à mecanização das operações agrícolas, nas diferentes culturas, representam entre 10 e $30 \%$ dos custos totais de produção, demonstrando a importância da escolha do equipamento mais adequado para o processo produtivo. VALENTE et al. (2011) elaboraram um programa de apoio à decisão para a determinação dos custos em unidades armazenadoras de grãos e verificaram que este trouxe flexibilidade ao suporte à decisão. QI et al. (2009) desenvolveram um programa computacional para uma análise estatística do uso da maquinaria agrícola em uma região da China e verificaram que o uso deste programa permitiu uma análise contínua mais eficiente ao longo do tempo. MERCANTE et al. (2010) elaboraram um programa computacional para a seleção e a estimativa do custo operacional de máquinas e implementos agrícolas, e verificaram que este pode fornecer ao usuário a melhor escolha da máquina, tanto do ponto de vista técnico, quanto do econômico. BAIO et al. (2004) desenvolveram um modelo por programação linear, para a seleção de pulverizadores agrícolas com base no menor custo operacional; entretanto, o modelo necessita de um programa computacional dedicado para analisar os dados, tonando-se uma ferramenta pouco útil para uso em tempo real pela Internet.

Os programas computacionais disponíveis até o momento não apresentam um modo de atualização contínua da base de dados ou do algoritmo de seleção. Assim, as atualizações são feitas por uma nova instalação no computador do usuário, levando a uma problemática da desatualização constante. $\mathrm{O}$ objetivo deste trabalho foi desenvolver um modelo de computador por programação linear em plataforma web, para seleção automatizada de conjuntos mecanizados agrícolas baseados no menor custo operacional.

\section{DESCRIÇÃO DO ASSUNTO}

\section{Estruturação e programação}

A programação foi desenvolvida utilizando a linguagem orientada a objeto em ASP.NET através do programa computacional Visual Studio Express, da Microsoft ${ }^{\circledR}$, o qual tem sua utilização livre para fins acadêmicos. O programa desenvolvido foi nomeado SELECT e contém os módulos de cadastros de usuários, fazenda, frota da fazenda, operações mecanizadas, cálculos das simulações e de relatórios. No módulo de cadastro de máquinas e implementos, foi implementado um banco de dados pelo uso da interface web onde foram gravadas as características técnicas das máquinas e implementos no Brasil como: marca, modelo, potência do motor (quando autopropelido), potência necessária (quando implemento), preço médio do mercado local (se é uma máquina nova ou usada), vida útil do equipamento, largura efetiva e velocidade da operação. 
A disponibilização do programa desenvolvido à comunidade foi realizada por um servidor dedicado ao programa SELECT. Este servidor foi cadastrado na rede web da Universidade Federal de Mato Grosso do Sul (UFMS) e possui um DNS fixo (http://www.maquinas.ufms.br). O programa SELECT não necessita de uma instalação prévia no computador do usuário, pois pode operar via Internet e por telefone celular com interface gráfica.

A seleção de máquinas pelo usuário foi realizada com base na análise operacional do planejamento de produção da fazenda, nas informações técnicas e econômicas das máquinas registradas pelo usuário e nas eficiências de campo operacionais cadastradas nas tabelas da base de dados ou provenientes da própria propriedade agrícola, quando este dado é inserido pelo usuário no programa.

\section{Embasamento técnico}

O código de programação foi desenvolvido de modo que a compatibilidade do conjunto mecanizado cadastrado pelo usuário seja testada conforme a potência exigida e a disponibilizada ao implemento, de tal forma que a potência disponibilizada seja maior que a exigida. As especificações técnicas de cada modelo fabricado pelos diversos fabricantes brasileiros foram obtidas de seus prospectos comerciais disponibilizados na Internet. A capacidade de campo operacional foi calculada a partir dos dados dimensionais de cada implemento e das estimativas de eficiência das operações, conforme MIALHE (1974), SILVEIRA et al. (2006), ASAE (2001) e HANNA (2011). Um dos parâmetros de extrema importância para a determinação da capacidade operacional de um conjunto mecanizado é a eficiência da operação em campo. Para BAIO (2011), no cálculo desta eficiência, leva-se em conta a porcentagem de tempo em que o conjunto mecanizado está efetivamente em atividade em campo, desconsiderando-se todos os tempos perdidos de reabastecimentos, deslocamentos e paradas por quebras. $\mathrm{O}$ autor ainda comenta que as tabelas clássicas utilizadas como fonte destas informações foram levantadas em talhões típicos dos EUA, tornando-se fonte de informação discutível para as condições brasileiras, como as informações apresentadas por ASAE (2001), quando não contrastadas com outras fontes, podendo esconder riscos de gasto desnecessário no dimensionamento ou falta de máquinas. O programa desenvolvido permite que o usuário cadastre uma eficiência operacional diferente daquela sugerida, de forma a adequar as condições intrínsecas do local, como sugerido por BAIO (2011). Para SILVEIRA et al. (2006), a eficiência média obtida nas operações de aração $(66,0 \%)$, gradagem $(62,1 \%)$, semeadura $(42,9 \%)$ e cultivo do milho $(60,5 \%)$ foi considerada baixa, quando comparada a valores obtidos de literatura, que relata uma eficiência entre 70 a $90 \%$ nessas operações.

Os custos com as máquinas e implementos foram divididos em custos fixos e variáveis. A metodologia utilizada para a seleção e custos operacionais da maquinaria agrícola está de acordo com MIALHE (1974), HUNT (1995), ASAE (2001), ASAE (2002) e BALASTREIRE (2007). Segundo OLIVEIRA \& MILAN (2001), é mais apropriado avaliar a vida útil de uma máquina em horas do que em anos. No programa SELECT, os preços das máquinas são informados pelo usuário, uma vez que varia sazonalmente com os preços das commodities, assim como nas regiões de venda. Caso o usuário não informe o preço como valor de entrada, o programa busca automaticamente o preço da máquina na base de dados do sistema, mas, nesse caso, para preservar as fontes dessa informação, os preços das máquinas cadastrados na base de dados não são mostrados. $\mathrm{O}$ acompanhamento do desempenho e os cálculos dos custos operacionais das máquinas agrícolas são fatores fundamentais para seu uso racional (OLIVEIRA \& MILAN, 2001).

Os custos fixos foram definidos como aqueles que não variam em relação à taxa de produção (MARTINS, 2009). São despesas contraídas da posse de algum bem e não são influenciadas pela quantidade de utilização do mesmo. Esses incluem as seguintes despesas: depreciação; juros; alojamento e seguro. A depreciação foi calculada em horas de acordo com o método linear, onde o valor da máquina é depreciado de um valor constante ao longo dos anos da vida útil, restando ao seu final o valor de sucata, em 10\% do valor inicial (BALASTREIRE, 2007). O programa foi desenvolvido com a capacidade de utilizar o valor inicial médio da máquina, proveniente das 
tabelas da base de dados (mantidas pelo seu administrador) ou pelo valor de mercado local, proveniente da inserção do preço inicial da máquina pelo usuário. Assim, o programa somente utiliza o valor médio da máquina, via base de dados do sistema, se o usuário não cadastrar a informação de preço inicial da máquina. Caso o usuário tenha cadastrado no programa a informação de uma máquina usada, inserindo o ano e o preço de mercado local, o programa calcula a depreciação deste a partir do ponto de depreciação atual, baseado na idade (horas) e na vida útil restante (horas) da máquina. Segundo OLIVEIRA \& MILAN (2001), os tratores agrícolas no Brasil têm vida útil média de 15 anos, diferindo das informações das tabelas técnicas da ASAE (2001). Contudo, em algumas situações críticas de uso da maquinaria agrícola no Brasil, como as dos tratores utilizados para o preparo de solo e plantio dentro do segmento cana, a vida útil dos tratores está ao redor de quatro anos, devido ao elevado uso, com mais de $3.000 \mathrm{~h} \mathrm{ano}^{-1}$. Assim, o algoritmo de cálculo da depreciação da máquina pelo programa computacional pôde considerar o valor real de uso por ano na propriedade, caso o usuário o tenha cadastrado, em uma tabela específica dentro do programa, junto aos dados cadastrados como financeiros. A taxa de juros é um componente do custo fixo, pois reflete o custo de oportunidade do uso do recurso sobre o investimento na aquisição da máquina agrícola. A taxa de juros é uma variável de entrada no programa, sendo sugerida automaticamente ao usuário a taxa de juros aplicada pelo programa Finame (BNDES, 2011), por ser a linha de crédito mais utilizada no Brasil para a compra de máquinas agrícolas; todavia, pode ser alterada pelo usuário. Os custos referentes ao seguro e ao alojamento foram adotados daqueles sugeridos por ASAE (2002), compondo $1 \%$ ao ano sobre o valor de aquisição da máquina.

Os custos variáveis dizem respeito aos dispêndios com fatores de produção variáveis, como combustíveis, mão de obra, manutenção preventiva e corretiva (MARTINS, 2009). Segundo OLIVEIRA \& MILAN (2001), estes custos atingem entre 10 e $20 \%$ do total do custo horário do maquinário. Para OLIVEIRA \& MILAN (2001) e BAIO et al. (2004), a taxa de reparo do maquinário agrícola é o fator que mais afeta o custo horário, seguido da taxa de depreciação. Dentre todos os custos da mecanização agrícola, os da manutenção preventiva e corretiva são altamente variáveis para um mesmo tipo de máquina e discrepantes entre os tipos de máquina, em função das inúmeras situações de uso (MERCANTE et al., 2010; ASAE, 2002; ASAE, 2001; OLIVEIRA \& MILAN, 2001). Vale ressaltar, também, que este custo é crescente ao longo dos anos de uso da máquina, ou seja, o desgaste de seus componentes eleva este custo. Assim, foi adotado um modelo linear para a estimativa dos custos da manutenção corretiva e preventiva, sugerida por ASAE (2002), e que foi dependente do tipo de máquina, do preço inicial e de sua vida útil. Os custos de mão de obra foram contabilizados pelo programa baseados na divisão do custo mensal do operador em relação às horas mensais referentes à operação. $\mathrm{O}$ valor referente ao salário do operador cadastrado pelo usuário foi multiplicado pelo fator 2,1, referente aos encargos trabalhistas sobre o salário médio de um operador de máquinas de uma empresa rural, conforme a CLT (Decreto Lei $\mathrm{n}^{\mathrm{o}}$ 5452 , de $1^{\circ}$ de maio de 1943). O custo da mão de obra para o conjunto mecanizado foi associado aos cálculos dos custos da fonte de potência, seja ela um trator, seja um autopropelido. O consumo de combustível de uma máquina agrícola (trator ou autopropelido) está diretamente relacionado à sua potência disponível e às condições operacionais, como tipo de implemento, profundidade de trabalho, teor de argila no solo, rotação do motor em regime de trabalho, dentre outros. Até mesmo duas unidades de um mesmo modelo de máquina agrícola, colocadas na mesma situação de regime de trabalho terão consumos diferentes. O consumo anual de diesel pode ser baseado em uma equação linear, relacionando a máxima potência disponível na tomada de potência do trator e um fator resultante da avaliação do consumo de tratores de rodas em pistas de teste (Nebraska Tractor Test Data). Assim, o consumo de combustível do trator ou do autopropelido em avaliação pelo programa foi estimado de acordo com ASAE (2002).

\section{Modelagem matemática por programação linear}

O modelo matemático de seleção do conjunto mecanizado pelo menor custo operacional é baseado em programação linear pelo Método Simplex e foi inserido no programa SELECT pelas eqs.(1); (2); (3); (4) e (5). A rotina de programação linear pelo Método Simplex em PHP 
(Hypertext Preprocessor), sugerida por MASSI (2011), foi adaptada e inserida na linguagem de programação em ASP.NET. Na eq.(1), ilustra-se a função da minimização dos custos.

$$
\text { Min } \mathrm{C}=\sum_{\mathrm{k}=1}^{\mathrm{n}} \sum_{\mathrm{l}=1}^{\mathrm{n}} \mathrm{x}_{\mathrm{k}, 1}\left(\operatorname{pin}_{\mathrm{k}} \frac{\left(\mathrm{chi}_{\mathrm{k}}+\mathrm{cht}_{1}\right)}{\mathrm{CCO}_{\mathrm{k}}}\right)
$$

em que,

Min C - minimização do custo operacional, $\mathrm{R} \$ \mathrm{ha}^{-1}$;

$\mathrm{x}_{\mathrm{k}, \mathrm{l}}$ - conjunto mecanizado formado pelo implemento k e o trator $\mathrm{l}$;

$\operatorname{pin}_{\mathrm{k}}$ - número de implementos necessários;

chi $\mathrm{k}_{\mathrm{k}}$ - custo horário total do implemento $\mathrm{k}, \mathrm{R} \$ \mathrm{~h}^{-1}$;

$\mathrm{cht}_{1}$ - custo horário total do trator $1, \mathrm{R} \$ \mathrm{~h}^{-1}$, e

$\mathrm{CCO}_{\mathrm{k}}$ - capacidade de campo operacional do implemento $\mathrm{k}$, ha $\mathrm{h}^{-1}$.

As restrições impostas ao modelo foram:

Número mínimo de conjuntos selecionados.

$$
\sum_{\mathrm{k}=1}^{\mathrm{n}} \sum_{\mathrm{l}=1}^{\mathrm{n}} \mathrm{x}_{\mathrm{k}, \mathrm{l}} \geq 1
$$

Número máximo de operadores disponíveis na propriedade.

$$
\sum_{\mathrm{k}=1}^{\mathrm{n}} \mathrm{x}_{\mathrm{k}, 1} \operatorname{pin}_{\mathrm{k}} \leq \mathrm{nm}
$$

Trator "virtual" somente pode ser selecionado quanto um autopropelido for selecionado.

$$
\sum_{\mathrm{k}=1}^{\mathrm{n}} \mathrm{x}_{\mathrm{kvirtual}, \text { virtual }}=0
$$

Restrição binária para o conjunto mecanizado trator e implemento.

$$
x_{k, l}=0 \text { ou } 1, \text { para } \mathrm{k}=1,2, \ldots, \mathrm{n} \mathrm{e} 1=1,2 \ldots, \mathrm{n}
$$

O cálculo do custo operacional mínimo $\left(\mathrm{R} \$ \mathrm{ha}^{-1}\right)$ é apresentado ao usuário em formato de relatórios. $\mathrm{O}$ módulo de resultados envolve os itens de estimativa do tempo disponível, estimativa do ritmo operacional e a estimativa do número de conjuntos mecanizados necessários, assim como a sumarização de todos os custos.

\section{Estudo de caso e validação}

Foi realizado um estudo de caso baseado em uma propriedade agrícola hipotética, situada na região de Chapadão do Sul-MS, para a validação do programa SELECT e aplicação prática do modelo. Simulou-se em uma área com cultivo de 386 ha de soja (Glycine max). Foram consideradas as seguintes atividades mecanizadas na área: uma aração, uma gradagem, uma aplicação de corretivo por cobertura, um plantio, duas pulverizações e uma colheita. Coube ao programa adequar a frota de acordo com as restrições dos Ritmos Operacionais ditados pelas condições das operações agrícolas, em seus tempos disponíveis.

A base de dados conta com tabelas que foram divididas em duas categorias: as tabelas base do sistema e as do usuário. As tabelas do sistema serviram de base para o usuário escolher, em uma lista de opções predefinidas, o parque de máquinas existente em sua propriedade. A tabela de implementos cadastrados possui 2.469 modelos disponibilizados no mercado brasileiro em 2011. Essa tabela relaciona os dados técnicos utilizados pelo programa para os cálculos do sistema 
mecanizado: categoria de implemento, tipo, marca, modelo, potência necessária para tração, ano de fabricação, preço, eficiência operacional, velocidade de trabalho, largura efetiva, tipo de operação e vida útil. Da mesma forma, a tabela de tratores possui 135 modelos encontrados no mercado brasileiro em 2011.

$\mathrm{Na}$ simulação de validação do programa, foram utilizados dois modelos de tratores e seis modelos de implementos. A seleção de máquinas autopropelidas é realizada pela inserção de implementos. Após a seleção dos implementos (Figura 1a), o usuário cadastra as informações financeiras relativas à área onde se situa a propriedade, como: preço local do combustível, taxa de juros anual (Finame), salário do operador (menos encargos), uso anual (horas.ano ${ }^{-1}$ ) do trator, da colhedora, dos implementos para o preparo de solo, semeadura, pulverização, cultivo e transporte.

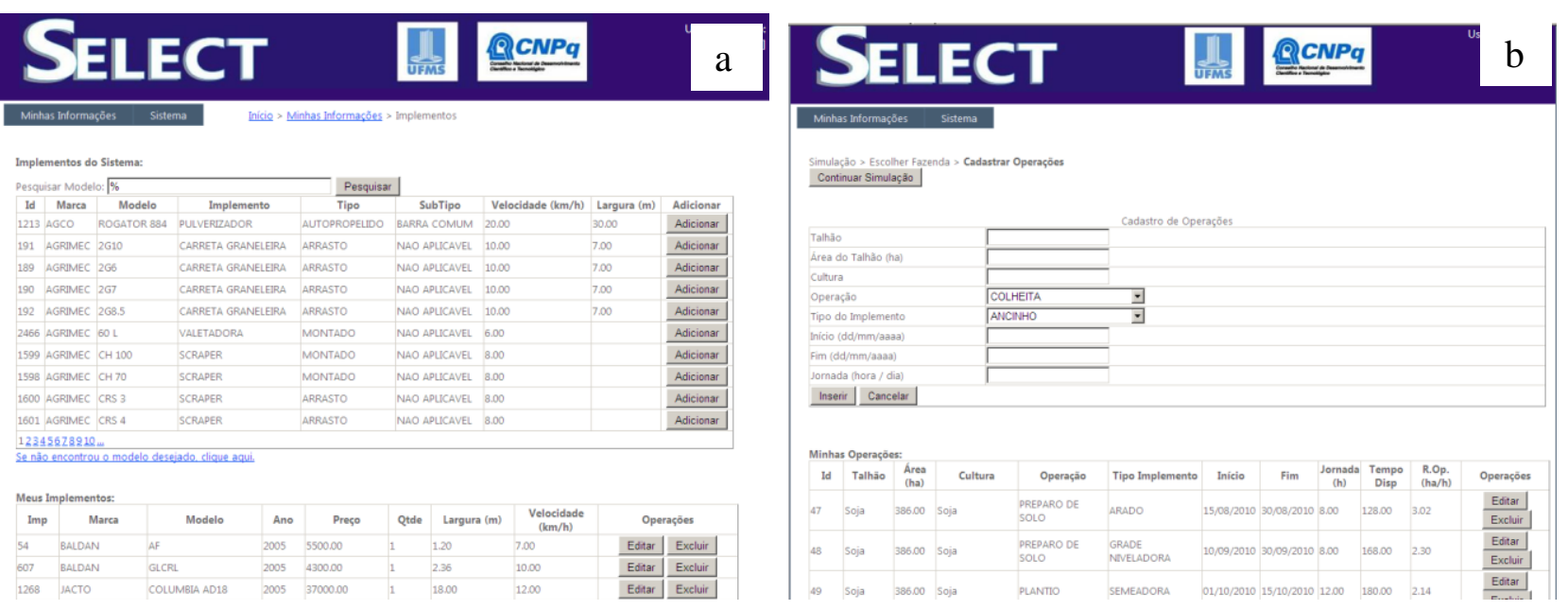

FIGURA 1. Tela de seleção de implementos (a) e de cadastro das operações mecanizadas (b). Screenshot of the implements selection (a) and registration of the mechanized activities (b).

Após a inclusão de informações financeiras, o usuário é convidado a cadastrar uma nova simulação. É pela simulação que todos os cálculos são realizados. Para cada simulação, é atribuída uma descrição e data, possibilitando o acesso a várias simulações para uma mesma propriedade, seja pelas variações de custos ou de condições técnicas, facilitando o gerenciamento da tomada de decisão sobre a aquisição ou o gerenciamento do parque de máquinas.

$\mathrm{Na}$ próxima etapa (Figura 1b), é realizado o cadastro das operações agrícolas, onde são atribuídos o nome do talhão, sua área (ha), as operação, o tipo de implemento utilizado em cada operação, o dia em que inicia a operação, o dia em que termina e a jornada de trabalho. As informações provenientes dos cálculos do Ritmo Operacional (RO, em ha $\mathrm{h}^{-1}$ ) para esta simulação de validação estão ilustradas na Figura 2. O programa calcula, automaticamente, quando a Capacidade de Campo Operacional ( $\mathrm{CCO}$, em ha $\left.\mathrm{h}^{-1}\right)$ do conjunto mecanização inserido não é capaz de atender à necessidade imposta pelo RO, retornando ao usuário um indicativo pela cor da linha correspondente a este cálculo, que possui uma margem de segurança de 10\%: adequação do conjunto mecanizado (verde) ou o conjunto mecanizado não atende às necessidades do RO (vermelho) ou o conjunto mecanizado possui uma CCO muito maior que a do RO (amarelo). O usuário pode inserir mais de um conjunto mecanizado para a realização da mesma operação, ou seja, é permitido que vários conjuntos mecanizados trabalhem na mesma operação e no mesmo talhão, como ocorre na prática. Este é um dos relatórios técnicos sobre a frota da propriedade, ilustrando sua capacidade técnica em realizar a operação dentro das condições estabelecidas. Pode-se observar que, para a simulação do plantio de soja em 386 ha, os implementos para o preparo do solo estão subdimensionados, assim como os implementos para a distribuição de adubo e a pulverização com o autopropelidos estão superdimensionados, cabendo ao usuário a tomada de uma decisão sobre a melhor adequação técnica de seu parque de máquinas. 


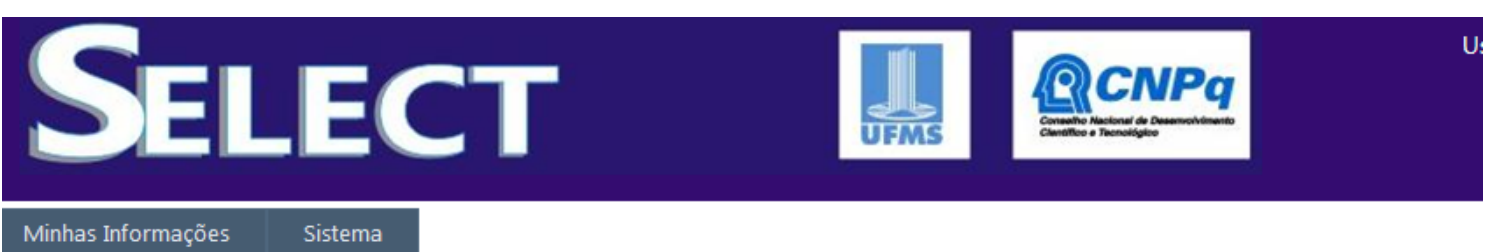

Simulação > Escolher Fazenda > Cadastrar Operações > Cadastrar Sistemas Mecanizados Continuar Simulação

\begin{tabular}{l|l|}
\multicolumn{2}{c}{ Cadastro do Sistema Mecanizado } \\
\cline { 2 - 3 } Operação & Operação: COLHEITA - Cultura: Soja - Talhão: Soja - Período: 05/01/2011 a 20/01/2011 \\
\hline Trator & MASSEY FERGUSON - 275 - 2005 \\
Implemento & BALDAN-AF - 2005 \\
\hline Inserir & Cancelar \\
\hline
\end{tabular}

\begin{tabular}{|c|c|c|c|c|c|c|c|c|}
\hline ID Talhão & Cultura & Operação & Tipo Imp & Implemento & Trator & R.Op. (ha/h) & CCO (ha/h) & Excluir \\
\hline 44 Soja & Soja & CULTIVO & CALCAREADORA & LANCER 1350 & 275 & 7.72 & 9.24 & Excluir \\
\hline 45 Soja & Soja & PREPARO DE SOLO & ARADO & AF & 785 & 3.22 & 0.71 & Excluir \\
\hline 46 Soja & Soja & PREPARO DE SOLO & GRADE NIVELADORA & GLCRL & 785 & 2.41 & 2.01 & Excluir \\
\hline 47 Soja & Soja & PLANTIO & SEMEADORA & PSM 132 ROTO & 785 & 2.30 & 2.27 & Excluir \\
\hline 48 Soja & Soja & PULVERIZACAO & PULVERIZADOR & COLUMBIA AD18 & 275 & 12.06 & 12.96 & Excluir \\
\hline 49 Soja & Soja & PULVERIZACAO & PULVERIZADOR & UNIPORT 2000 PLUS & AUTOPROPELIDO & 9.65 & 29.40 & Excluir \\
\hline 50 Soja & Soja & COLHEITA & COLHEDORA & TC 57 & AUTOPROPELIDO & 2.57 & 2.34 & Excluir \\
\hline
\end{tabular}

FIGURA 2. Ilustração da associação do conjunto no sistema mecanizado. Screenshot illustration of the mechanized system association.

O relatório da comparação dos custos apresenta não somente os custos horários do trator e dos implementos para cada operação agrícola, como também os custos operacionais, utilizados para o planejamento agrícola. O programa calcula os custos horários e os custos por unidade de área para cada operação cadastrada dentro da simulação, possibilitando a impressão e a comparação com outras simulações.

Na Figura 3, ilustra-se a sugestão do implemento com um menor custo operacional para a distribuição de calcário referente à simulação nos 386 ha. Foi realizada uma modelagem matemática por programação linear para cada operação mecanizada, sugerindo uma listagem de implementos que atendem ao ritmo operacional, em ordem crescente pelo custo operacional do conjunto mecanizado, facilitando ao usuário a tomada de uma decisão. 
Selecione uma operação

\begin{tabular}{|l|l|l|}
\hline \multicolumn{1}{|c|}{ Id } & \multicolumn{1}{|c|}{ Operação } & Sugestão de Implemento \\
\hline 105 & Período: $10 / 08 / 2011$ a 15/08/2011 - Operação: CULTVO - Cultura: Soja - Talhão: 1 & Sugerir \\
\hline 106 & Período: $15 / 08 / 2011$ a 30/08/2011 - Operação: PREPARO DE SOLO - Cultura: Soja - Talhão: 1 & Sugerir \\
\hline 107 & Período: 10/09/2011 a 30/09/2011 - Operação: PREPARO DE SOLO - Cultura: Soja - Talhão: 1 & Sugerir \\
\hline 108 & Período: 01/10/2011 a 15/10/2011 - Operação: PLANTIO - Cultura: Soja - Talhão: 1 & $\underline{\text { Sugerir }}$ \\
\hline 109 & Período: 01/11/2011 a 05/11/2011 - Operação: PULVERIZACAO - Cultura: Soja - Talhão: 1 & $\underline{\text { Sugerir }}$ \\
\hline 110 & Período: $25 / 11 / 2011$ a 30/11/2011 - Operação: PULVERIZACAO - Cultura: Soja - Talhão: 1 & Sugerir \\
\hline 111 & Periodo: 09/12/2011 a 12/12/2011 - Operação: PULVERIZACAO - Cultura: Soja - Talhão: 1 & Sugerir \\
\hline 112 & Período: 05/01/2012 a 15/01/2012 - Operação: COLHEITA - Cultura: Soja - Talhão: 1 & Sugerir \\
\hline
\end{tabular}

Implementos Sugeridos

\begin{tabular}{|c|c|c|c|c|c|c|c|c|}
\hline ID & Marca & Modelo & Implemento & Tipo & SubTipo & Largura & $\begin{array}{l}\mathrm{CCO} \\
(\mathrm{ha} / \mathrm{h})\end{array}$ & $\begin{array}{l}\text { Custo por Área } \\
\text { (R\$/ha) }\end{array}$ \\
\hline 139 & PINHEIRO & DCP 600 & CALCAREADORA & MONTADO & $\begin{array}{l}\mathrm{NAO} \\
\text { APLICAVEL }\end{array}$ & 16,00 & 12,32 & 1,04 \\
\hline 140 & SEMBRA & 1300.P AH & CALCAREADORA & MONTADO & $\begin{array}{l}\mathrm{NAO} \\
\text { APLICAVEL }\end{array}$ & 12,00 & 9,24 & 2,10 \\
\hline 110 & JAN & LANCER 600.PENDULA & CALCAREADORA & MONTADO & $\begin{array}{l}\mathrm{NAO} \\
\text { APLICAVEL }\end{array}$ & 12,00 & 9,24 & 2,34 \\
\hline 108 & JAN & $\begin{array}{l}\text { LANCER } 600 \\
\text { MONODIS }\end{array}$ & CALCAREADORA & MONTADO & $\begin{array}{l}\mathrm{NAO} \\
\text { APLICAVEL }\end{array}$ & 12,00 & 9,24 & 2,80 \\
\hline
\end{tabular}

FIGURA 3. Ilustração do relatório automatizado do conjunto mecanizado com um menor custo operacional. Screenshot illustration of the automatized report about the mechanized system with the lowest operational cost.

\section{CONCLUSÕES}

O modelo matemático desenvolvido, baseado em programação linear pelo método SIMPLEX, selecionou conjuntos mecanizados com menores custos operacionais.

O programa proporcionou ao usuário uma seleção racional via Internet de conjuntos mecanizados e o estudo econômico do uso das máquinas e implementos, sem a necessidade da instalação de programas dedicados no computador.

Além do uso técnico, o programa apresentou-se como uma ferramenta que poderá ser utilizada na fixação do conteúdo teórico do plano de ensino em disciplinas dos cursos em Ciências Agrárias.

\section{AGRADECIMENTOS}

Ao $\mathrm{CNPq}$, pelo suporte financeiro, e a todos os participantes do projeto SELECT que auxiliaram na implementação do banco de dados de máquinas disponíveis no mercado nacional, destacando: Luan A. Matos; Moacyr T. Maekawa Jr.; Rafael S. Faraun; Bruno R. Castilho; Anderson L. Petternan; Marcos F. Oliveira; e Sherithon M. Paula.

\section{REFERÊNCIAS}

ASAE. Agricultural machinery management data - D497.4. St. Joseph: ASAE Standards, 2001. p.362-369.

ASAE. Agricultural machinery management - EP496.2. St. Joseph: ASAE Standards, 2002. p.366371.

BAIO, F.H.R. Os perigos no planejamento da frota agrícola. Agrianual, São Paulo, v.16, p.103, 2011. 
BAIO, F.H.R.; ANTUNIASSI, U.R.; BALASTREIRE, L.A.; CAIXETA FILHO, J.V. Modelo de programação linear para seleção de pulverizadores agrícolas de barras. Engenharia Agrícola, Jaboticabal, v.24, n.2, p.355-363, 2004.

BALASTREIRE, L.A. Máquinas agrícolas. 3.ed. Piracicaba, 2007. 307 p.

BNDES. Finame. Disponível em: <http://www.bndes.gov.br/SiteBNDES/bndes/bndes_pt/ Institucional/Apoio_Financeiro/Produtos/FINAME_Maquinas_e_Equipamentos/> Acesso em: 5 nov. 2011.

BORNSTEIN, C.T.; MACULAN, N.; PASCOAL, M.; PINTO, L.L. Multiobjective combinatorial optimization problems with a cost and several bottleneck objective functions: an algorithm with reoptimization. Computers \& Operations Research, Oxford, v.39, n.9, p.1.969-1.976, 2011.

CENTENO, A.S.; KAERCHER, D. Custo operacional das máquinas agrícolas. Agrianual, São Paulo, v.15, p.113-116, 2010.

HANNA, M. Estimating the field capacity of farm machines. Disponível em:

<http://www.extension.iastate.edu/agdm/crops/pdf/a3-24.pdf>. Acesso em: 5 nov. 2011.

HUNT, D. Farm power and machinery management. Ames: Iowa State University Press, 1995. $363 \mathrm{p}$.

MARTINS, E. Contabilidade de custos. 9.ed. São Paulo: Atlas, 2009. 370 p.

MASSI, G. Implementazione del metodo simplesso in PHP. Disponível em: <http://gim.altervista.org/ro/>. Acesso em: 5 nov. 2011.

MERCANTE, E.; SOUZA, E.D.; JOHANN, J.A.; GABRIEL FILHO, A.; URIBE-OPAZO, M. Praprag - software para planejamento racional de máquinas agrícolas. Engenharia Agrícola, Jaboticabal, v.30, n.2, p.322-333, 2010.

MIALHE, L.G. Manual de mecanização agrícola. São Paulo: Agronômica Ceres, 1974. 301 p.

OLIVEIRA, M.D.M.; MILAN, M. Custo operacional e ponto de renovação de tratores agrícolas de pneus: avaliação de uma frota. In: CONGRESSO BRASILEIRO DE ENGENHARIA AGRÍCOLA, 30., 2001, Foz do Iguaçu. Anais... Jaboticabal: Associação Brasileira de Engenharia Agrícola, 2001. 1 CD-ROM.

QI, X.; LI, H.; YIN, W. Development of the farm machinery statistical management system based on web. In: LI, D.; CHUNJIANG, Z. (Org). Computer and computing technologies in agriculture. Boston: Springer, 2009, v.2, p.1.309-1.317.

SILVEIRA, G.M. da; YANAI, K.; KURACHI, S.A.H. Determinação da eficiência de campo de conjuntos de máquinas convencionais de preparo do solo, semeadura e cultivo. Revista Brasileira de Engenharia Agrícola e Ambiental, Campina Grande, v.10, n.1, p.220-224, 2006.

VALENTE, D.S.M.; QUEIROZ, D.M.; CORRÊA, P.C.C.; SILVA, L.C.; VALE, S.M.L.R. A decision support system for cost determination in grain storage facility operations. Engenharia Agrícola, Jaboticabal, v.31, n.4, p.735-744, 2011. 